\title{
On the Shear Buckling of Clamped Narrow Rectangular Orthotropic Plates
}

\author{
Seyed Rasoul Atashipour and Ulf Arne Girhammar \\ Department of Civil, Environmental and Natural Resources Engineering, Division of Structural and \\ Construction Engineering-Timber Structures, Luleå University of Technology, 97187 Luleå, Sweden
}

Correspondence should be addressed to Seyed Rasoul Atashipour; rasoul.atashipour@ltu.se

Received 19 October 2015; Accepted 29 October 2015

Academic Editor: Francesco Tornabene

Copyright ( 2015 S. R. Atashipour and U. A. Girhammar. This is an open access article distributed under the Creative Commons Attribution License, which permits unrestricted use, distribution, and reproduction in any medium, provided the original work is properly cited.

\begin{abstract}
This paper deals with stability analysis of clamped rectangular orthotropic thin plates subjected to uniformly distributed shear load around the edges. Due to the nature of this problem, it is impossible to present mathematically exact analytical solution for the governing differential equations. Consequently, all existing studies in the literature have been performed by means of different numerical approaches. Here, a closed-form approach is presented for simple and fast prediction of the critical buckling load of clamped narrow rectangular orthotropic thin plates. Next, a practical modification factor is proposed to extend the validity of the obtained results for a wide range of plate aspect ratios. To demonstrate the efficiency and reliability of the proposed closed-form formulas, an accurate computational code is developed based on the classical plate theory (CPT) by means of differential quadrature method (DQM) for comparison purposes. Moreover, several finite element (FE) simulations are performed via ANSYS software. It is shown that simplicity, high accuracy, and rapid prediction of the critical load for different values of the plate aspect ratio and for a wide range of effective geometric and mechanical parameters are the main advantages of the proposed closed-form formulas over other existing studies in the literature for the same problem.
\end{abstract}

\section{Introduction}

The shear buckling analysis of clamped composite plates is of great importance in design of many types of engineering structures. Unlike the problem of normal buckling of plates, the shear buckling problem of plates is mathematically described by differential equations having a term with oddorder of derivatives with respect to each of the planar spatial coordinates. Therefore, their governing equations cannot be solved exactly. Such problems are almost always analysed and solved using different numerical approaches. Apart from the loading type, clamped boundary conditions at all plate edges make the problem more difficult for finding an exact analytical solution.

During the past decades, many investigators have studied the shear buckling problem of rectangular plates. One of the first efforts dealing with shear buckling analysis of clamped isotropic plates with finite dimensions can be attributed to Budiansky and Conner [1] using Lagrangian multiplier method. A useful review of the studies on the shear buckling of both isotropic and orthotropic plates was presented by Johns [2]. Shear buckling analysis of antisymmetric cross ply, simply supported rectangular plates was carried out by Hui [3] using Galerkin procedure. Kosteletos [4] studied shear buckling response of laminated composite rectangular plates with clamped edges using Galerkin method. Biggers and Pageau [5] computed shear buckling loads of both uniform and composite tailored plates using finite element method. Xiang et al. [6] employed pb-2 Rayleigh-Ritz approach to obtain critical shear loads of simply supported skew plates. Loughlan [7] studied the shear buckling of thin laminated composite plates and examined the effect of bend-twist coupling on their behaviour using a finite strip procedure. Lopatin and Korbut [8] utilized the finite difference method to investigate the shear buckling of thin clamped orthotropic plates. Shufirn and Eisenberger [9] analysed the buckling of thin plates under combined shear and normal compressive loads using the multiterm extended Kantorovich method. 
The shear buckling load of rectangular composite plates consisting of concentric rectangular layups was investigated by Papadopoulos and Kassapoglou [10] by means of a Rayleigh-Ritz approach. Wu et al. [11] calculated the critical shear buckling loads of rectangular plates by the extended spline collocation method (SCM). Uymaz and Aydogdu [12] carried out the shear buckling analysis of functionally graded plates for various boundary conditions based on the Ritz method. Shariyat and Asemi [13] performed a nonlinear elasticity-based analysis for the shear buckling of rectangular orthotropic functionally graded (FG) plates surrounded by elastic foundations using a cubic B-spline finite element approach.

Evidently, all the above-mentioned numerical studies have some deficiencies like convergence difficulties and being time-consuming compared to analytical and closedform solutions. Therefore, it is not easy and time-efficient to predict the critical shear buckling loads and investigate the effect of various parameters by the use of numerical solution approaches. To the best of authors' knowledge, no closed-form solution can be found in the literature for the shear buckling of composite rectangular plates with finite dimensions. To fill this apparent void, the present work is carried out to provide efficient and reliable explicit formulas for rapid prediction of the fundamental critical shear buckling loads of clamped orthotropic rectangular plates. The range of validity of the proposed closed-form formulas is extended by introducing a practical modification factor. Also, in order to demonstrate the efficiency and reliability of the proposed closed-form formulas, an accurate computational code is developed by means of differential quadrature method (DQM) for comparison purposes. Moreover, several finite element (FE) simulations are performed via ANSYS software.

This paper is only devoted to a principle study of the shear buckling behavior and, for illustration, is applied to a laminated veneer lumber (LVL) panel. Other failure modes, such as the shear strength, are not included in the analysis.

\section{Definition of the Problem and Governing Equations}

Consider a clamped narrow rectangular orthotropic plate of length $a$, width $b$, and thickness $t$, subjected to a uniformly distributed shear load per length $S_{x y}$ (Figure 1). The coordinates system is shown in the figure. We employ the classical plate theory (CPT) of Kirchhoff to study the shear buckling of thin plates. The governing equation of CPT for the orthotropic plates is expressed as

$$
\begin{aligned}
& D_{11} \frac{\partial^{4} w}{\partial x^{4}}+2\left(D_{12}+2 D_{33}\right) \frac{\partial^{4} w}{\partial x^{2} \partial y^{2}}+D_{22} \frac{\partial^{4} w}{\partial y^{4}} \\
& \quad=2 S_{x y} \frac{\partial^{2} w}{\partial x \partial y}
\end{aligned}
$$

where $w$ is transverse displacement, and $D_{i j}$ are stiffness coefficients of orthotropic materials and are defined as follows:

$$
\begin{aligned}
& D_{11}=\frac{E_{1} t^{3}}{12\left(1-\nu_{12} \nu_{21}\right)}, \\
& D_{12}=\frac{\nu_{21} E_{1} t^{3}}{12\left(1-\nu_{12} \nu_{21}\right)}, \\
& D_{22}=\frac{E_{2} t^{3}}{12\left(1-\nu_{12} \nu_{21}\right)}, \\
& D_{33}=\frac{1}{12} G_{12} t^{3}
\end{aligned}
$$

in which $E_{1}$ and $E_{2}$ are modulus of elasticity of orthotropic material in $x$ and $y$ directions, respectively; $G_{12}$ is the in-plane shear modulus and $v_{i j}$ are the Poisson's ratios.

The plate is assumed to be fully clamped. Thus, the following boundary conditions should be considered at the plate edges:

$$
\begin{aligned}
\left.w\right|_{x=0, a} & =0, \\
\left.w\right|_{y=0, b} & =0, \\
\left.\frac{\partial w}{\partial x}\right|_{x=0, a} & =0, \\
\left.\frac{\partial w}{\partial y}\right|_{y=0, b} & =0 .
\end{aligned}
$$

\section{An Efficient Closed-Form Solution}

As mentioned earlier, no exact analytical approach exists for the problem of shear buckling of rectangular orthotropic plates, not only due to the loading type, but also because of the fully clamped boundary conditions. The computational numerical approaches are usually time-consuming for obtaining the results with adequate accuracy. Therefore, it is reasonable to find an efficient method for predicting the critical loads. Timoshenko and Gere [14] presented an approximate solution for the shear buckling of narrow rectangular plates with the limitation of simply supported boundary conditions and isotropic material. We start by extending the method for the orthotropic narrow rectangular plates with clamped boundary conditions. To this end, we consider the following expression for the transverse displacement of the buckled plate:

$$
w(x, y)=\left[1-\cos \left(\frac{2 \pi}{b} y\right)\right] \cdot \sin \left[\frac{\pi}{s}(x-\alpha y)\right]
$$

where $s$ and $\alpha$ represent the length of half-waves of the buckled plate and the slope of the nodal lines. Clearly, (4) satisfies the clamped edge conditions at the long edges $y=$ $0, b$. However, this approximate approach is not capable of satisfying the clamped boundary conditions at the two short 


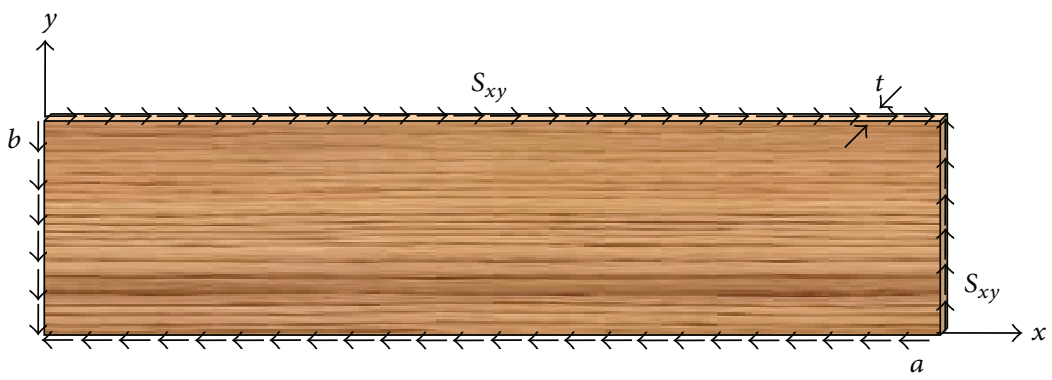

FIGURE 1: Geometric configuration and coordinate system of a narrow rectangular orthotropic plate with fully clamped edges subjected to a uniformly distributed shear load.

edges. The work done by the external forces and the strain energy during the buckling of the plate are defined by

$$
\begin{aligned}
\Delta T & =-S_{\text {cr }} \int_{0}^{2 s} \int_{0}^{b} \frac{\partial w}{\partial x} \frac{\partial w}{\partial y} d x d y \\
\Delta U & =\frac{1}{2} \int_{0}^{2 s} \int_{0}^{b}\left[D_{11}\left(\frac{\partial^{2} w}{\partial x^{2}}\right)^{2}+D_{22}\left(\frac{\partial^{2} w}{\partial y^{2}}\right)^{2}\right. \\
& +2 D_{12}\left(\frac{\partial^{2} w}{\partial x^{2}}\right)\left(\frac{\partial^{2} w}{\partial y^{2}}\right) \\
& \left.+4 D_{33}\left(\frac{\partial^{2} w}{\partial x \partial y}\right)^{2}\right] d x d y .
\end{aligned}
$$

By substituting the proposed form of the transverse displacement from (4) into (5) and equating the work of external forces to the strain energy (i.e., $\Delta T=\Delta U$ ), a closed-form formula is obtained for the critical buckling load as

$$
\begin{aligned}
S_{\mathrm{cr}} & =\frac{\pi^{2} s^{2}}{6 b^{4} \alpha}\left\{16 D_{22}\right. \\
& +8\left[\left(D_{12}+2 D_{33}\right)+3 D_{22} \alpha^{2}\right]\left(\frac{b}{s}\right)^{2} \\
& \left.+3\left[D_{11}+2\left(D_{12}+2 D_{33}\right) \alpha^{2}+D_{22} \alpha^{4}\right]\left(\frac{b}{s}\right)^{4}\right\} .
\end{aligned}
$$

The obtained formula for the critical buckling load should be minimized with respect to the unassigned parameters $s$ and $\alpha$. To this end, we differentiate (6) one time with respect to $s$ and then with respect to $\alpha$. It is easy to show that the resulted set of algebraic equations can be represented as

$$
\begin{aligned}
& 3 D_{11}+4\left(D_{12}+2 D_{33}\right)\left(\frac{s}{b}\right)^{2}-12 D_{22}\left(\frac{s}{b}\right)^{2} \alpha^{2} \\
& -3 D_{22} \alpha^{4}=0 \\
& 3 D_{11}+6\left(D_{12}+2 D_{33}\right) \alpha^{2}+3 D_{22} \alpha^{4} \\
& -16 D_{22}\left(\frac{s}{b}\right)^{4}=0 .
\end{aligned}
$$

Exact solution of the above set of equations can be represented in the form

$$
\begin{aligned}
\alpha & =\frac{\sqrt{2}}{2} \\
& \cdot \sqrt{\sqrt{\frac{\delta_{2}\left(7 \delta_{1}-19 \delta_{2}^{2} / 3\right)}{\sqrt{\delta_{3}+\delta_{4} / \ell+\ell / 3}}+2 \delta_{3}-\frac{\delta_{4}}{\ell}-\frac{\ell}{3}}+\sqrt{\delta_{3}+\frac{\delta_{4}}{\ell}+\frac{\ell}{3}}-\delta_{2},}
\end{aligned}
$$

where

$$
\ell=\sqrt[3]{\delta_{5}+\sqrt{\delta_{5}^{2}-27 \delta_{4}^{3}}}
$$

Also, the parameter $s$ is expressed in terms of $\alpha$ as follows:

$$
s=\frac{b}{2} \sqrt[4]{3\left(\delta_{1}+2 \delta_{2} \alpha^{2}+\alpha^{4}\right)} .
$$

In (8) through (10), the coefficients $\delta_{i}(i=1,2, \ldots, 5)$ are defined as

$$
\begin{aligned}
\delta_{1}= & \frac{D_{11}}{D_{22}}, \\
\delta_{2}= & \frac{\widetilde{D}_{12}}{D_{22}}, \\
\delta_{3}= & -\frac{5}{3}\left(\frac{D_{11}}{D_{22}}\right)+\frac{20}{9}\left(\frac{\widetilde{D}_{12}}{D_{22}}\right)^{2}, \\
\delta_{4}= & \frac{1}{12}\left(\frac{D_{11}}{D_{22}}\right)^{2}-\frac{7}{18}\left(\frac{D_{11}}{D_{22}}\right)\left(\frac{\widetilde{D}_{12}}{D_{22}}\right)^{2} \\
& +\frac{49}{108}\left(\frac{\widetilde{D}_{12}}{D_{22}}\right)^{4}, \\
\delta_{5}= & \frac{485}{8}\left(\frac{D_{11}}{D_{22}}\right)^{3}-\frac{587}{8}\left(\frac{D_{11}}{D_{22}}\right)^{2}\left(\frac{\widetilde{D}_{12}}{D_{22}}\right)^{2} \\
& +\frac{293}{24}\left(\frac{D_{11}}{D_{22}}\right)\left(\frac{\widetilde{D}_{12}}{D_{22}}\right)^{4}+\frac{181}{216}\left(\frac{\widetilde{D}_{12}}{D_{22}}\right)^{6}
\end{aligned}
$$

in which

$$
\widetilde{D}_{12}=D_{12}+2 D_{33}
$$


For the isotropic case, $\delta_{1}=\delta_{2}=1$ and, consequently, the parameters $\alpha$ and $s$ from (8)-(11) are reduced to

$$
\begin{aligned}
& \alpha=\frac{1}{\sqrt[4]{3}}, \\
& s=\frac{b}{2} \sqrt{1+\sqrt{3}} .
\end{aligned}
$$

Therefore, the critical buckling load from (6) is reduced to

$$
S_{\mathrm{cr}}=\frac{8}{3} \sqrt[4]{3}(\sqrt{3}+1) \frac{\pi^{2} D}{b^{2}} .
$$

It is worth to rewrite the obtained closed-form formula in the conventional form as follows:

$$
S_{\mathrm{cr}}=k_{s} \frac{\pi^{2} D_{11}}{b^{2}}
$$

Therefore, the dimensionless coefficient $k_{s}$ is represented in the form

$$
k_{s}=\frac{4}{3 \delta_{1} \alpha}\left\{\sqrt{3\left(\delta_{1}+2 \delta_{2} \alpha^{2}+\alpha^{4}\right)}+\delta_{2}+3 \alpha^{2}\right\}
$$

in which the coefficients $\alpha, \ell$, and $\delta_{i}(i=1,2, \ldots, 5)$ are defined by (8), (9), and (11).

It will be shown that the obtained closed-form formulas accurately predict the critical buckling load of a narrow rectangular orthotropic plate in shear with clamped edges. Apparently, the accuracy of the obtained closed-form formulas decreases when the plate aspect ratio decreases. To enhance the validity range of the obtained formulas for lower values of the plate aspect ratio and generalize them, we propose a simple practical modification factor $\left(C_{\mathrm{mf}}\right)$ to be multiplied by the dimensionless coefficient $k_{s}$ in the form:

$$
C_{\mathrm{mf}}=1+\frac{3 / \bar{E}+1}{4\left[(a / b)^{4}+1\right]}
$$

In the next section, a differential quadrature (DQ) code is developed for comparison purposes to prove the high accuracy of the proposed closed-form formulas for predicting the critical buckling load of the rectangular orthotropic plates in shear with clamped edges. Evidently, fast and easy prediction of the critical buckling load is the main advantage of the obtained closed-form formulas over existing studies in the literature based on time-consuming numerical approaches.

\section{Differential Quadrature Solution}

The differential quadrature method (DQM), as an appropriate method among various numerical solution approaches, has been mostly utilized by scientists for the eigen-buckling analysis of composite rectangular plates under in-plane normal compressive loads (e.g., see [15-18]). Here, we employ this methodology to solve the problem of shear buckling of clamped thin composite plates. To this end, we define the transverse displacement $w$ as a multipolynomial through discretized points $W_{i, j}=w\left(x_{i}, y_{j}\right)$ in the domain:

$$
w(x, y)=\sum_{i=1}^{N_{x}} \sum_{j=1}^{N_{y}} W_{i, j} f_{i}(x) g_{j}(y),
$$

where $N_{x}$ and $N_{y}$ are the number of grid points in $x$ and $y$ directions, respectively, and the Lagrange interpolation polynomials $f_{i}(x)$ and $g_{j}(y)$ are defined in the form

$$
\begin{aligned}
& f_{i}(x)=\prod_{k=1, k \neq i}^{N_{x}} \frac{x-x_{k}}{x_{i}-x_{k}}, \\
& g_{j}(y)=\prod_{k=1, k \neq j}^{N_{y}} \frac{y-y_{k}}{y_{i}-y_{k}} .
\end{aligned}
$$

It is assumed that the following equations are satisfied for the function $w(x, y)$ and its derivatives [19]:

$$
\begin{aligned}
w_{x}^{(n)}\left(x_{i}, y_{j}\right)= & \sum_{k=1}^{N_{x}} c_{i k}^{(n)} W_{k, j}, \\
& i=1,2, \ldots, N_{x} ; n=1,2, \ldots, N_{x}-1, \\
w_{y}^{(m)}\left(x_{i}, y_{j}\right)= & \sum_{k=1}^{N_{y}} \bar{c}_{j k}^{(m)} W_{i, k}, \\
& j=1,2, \ldots, N_{y} ; m=1,2, \ldots, N_{y}-1,
\end{aligned}
$$

where $c$ and $\bar{c}$ are weighting coefficients in the DQM for differentiation of $w$ with respect to $x$ of order $n$ and $y$ of order $m$, respectively. Details on calculations of the weighting coefficients, according to Shu's general approach [19], are given in Appendix.

Substituting (20) into (1) results in the discretized governing equation as follows:

$$
\begin{gathered}
D_{11} \sum_{k=1}^{N_{x}} c_{i k}^{(4)} W_{k, j}+2\left(D_{12}+2 D_{33}\right) \sum_{k_{1}=1}^{N_{x}} \sum_{k_{2}=1}^{N_{y}} c_{i k_{1}}^{(2)} \bar{c}_{j k_{2}}^{(2)} W_{k_{1}, k_{2}} \\
+D_{22} \sum_{k=1}^{N_{y}} \bar{c}_{j k}^{(4)} W_{i, k}=2 S_{x y} \sum_{k_{1}=1}^{N_{x}} \sum_{k_{2}=1}^{N_{y}} c_{i k_{1}}^{(1)} \bar{c}_{j k_{2}}^{(1)} W_{k_{1}, k_{2}} .
\end{gathered}
$$

To obtain accurate results, the distribution of the grid points should be denser at the edge-zones. Thus, an appropriate grid point distribution pattern is used as

$$
\begin{aligned}
& \frac{x_{i}}{a}=\frac{1}{2}\left[1-\cos \left(\frac{i-1}{N_{x}-1} \pi\right)\right], \quad i=1,2, \ldots, N_{x}, \\
& \frac{y_{j}}{b}=\frac{1}{2}\left[1-\cos \left(\frac{j-1}{N_{y}-1} \pi\right)\right], \quad j=1,2, \ldots, N_{y},
\end{aligned}
$$

where $x_{i}$ and $y_{j}$ are the coordinates of $i$ th and $j$ th grid points, respectively. Figure 2 shows the mesh distribution on a narrow rectangular domain based on (22). 


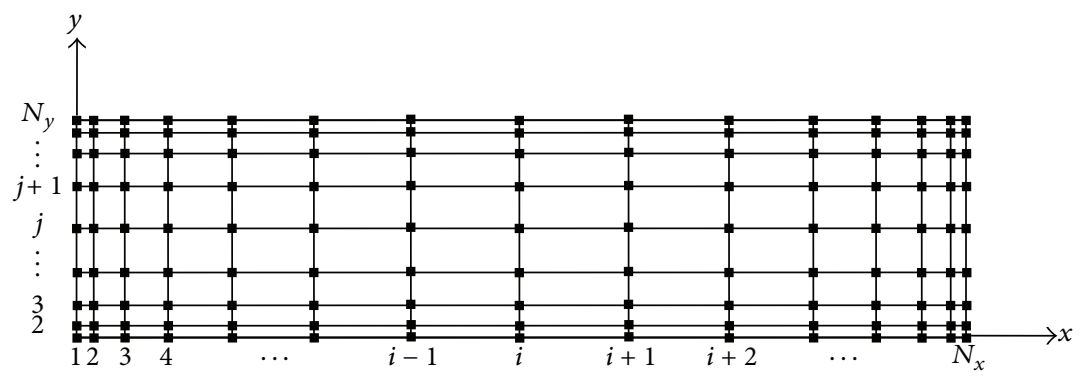

FIGURE 2: Illustration of the DQ meshed narrow rectangular plate.

The boundary conditions (3a) and (3b) can be rewritten in the form

$$
\begin{aligned}
W_{1, j}=0, & \text { at } x=0, \\
W_{N_{x}, j}=0, & \text { at } x=a, \\
W_{i, 1}=0, & \text { at } y=0, \\
W_{i, N_{y}}=0, & \text { at } y=b, \\
\sum_{k=1}^{N_{x}} c_{1, k}^{(1)} W_{k, j}=0, & \text { at } x=0, \\
\sum_{k=1}^{N_{x}} c_{N_{x}, k}^{(1)} W_{k, j}=0, & \text { at } x=a, \\
\sum_{k=1}^{N_{y}} \bar{c}_{1, k}^{(1)} W_{i, k}=0, & \text { at } y=0, \\
\sum_{k=1}^{N_{y}} \bar{c}_{N_{y}, k}^{(1)} W_{i, k} & =0, \quad \text { at } y=b .
\end{aligned}
$$

Clearly, (23a) and (23b) can be easily satisfied at the four edges of the plate. However, (24a) and (24b) cannot be directly substituted into (21). This difficulty can be easily overcome by using a simple method described by Shu and Du [20]. Based on this approach, the two equations (24a) are coupled to give two solutions $W_{2, j}$ and $W_{N_{x}-1, j}$ as follows:

$$
\begin{aligned}
W_{2, j} & =\frac{1}{\alpha_{0}} \sum_{k=3}^{N_{x}-2} \beta_{k} W_{k, j}, \\
W_{N_{x}-1, j} & =\frac{1}{\alpha_{0}} \sum_{k=3}^{N_{x}-2} \gamma_{k} W_{k, j},
\end{aligned}
$$

Using a similar method, the two equations (24b) can be coupled to give two solutions for $W_{i, 2}$ and $W_{i, N_{y}-1}$ :

$$
\begin{aligned}
W_{i, 2} & =\frac{1}{\bar{\alpha}_{0}} \sum_{k=3}^{N_{y}-2} \bar{\beta}_{k} W_{i, k}, \\
W_{i, M-1} & =\frac{1}{\bar{\alpha}_{0}} \sum_{k=3}^{N_{y}-2} \bar{\gamma}_{k} W_{i, k}, \\
& i=3,4, \ldots, N_{x}-2 .
\end{aligned}
$$

The coefficients $\alpha_{0}, \beta_{k}, \gamma_{k}, \bar{\alpha}_{0}, \bar{\beta}_{k}$ and $\bar{\gamma}_{k}$, in (25) and (26), are defined in Appendix.

For the points near the four corners, $W_{2,2}, W_{N_{x}-1,2}$, $W_{2, N_{y}-1}$, and $W_{N_{x}-1, N_{y}-1}$ can be determined by coupling (24a) and $(24 \mathrm{~b})$ in the following form:

$$
\begin{array}{r}
W_{2,2}=\frac{1}{\alpha_{0} \bar{\alpha}_{0}} \sum_{k_{1}=3}^{N_{x}-2} \sum_{k_{2}=3}^{N_{y}-2} \beta_{k} \bar{\beta}_{k} W_{k_{1}, k_{2}}, \\
W_{N_{x}-1,2}=\frac{1}{\alpha_{0} \bar{\alpha}_{0}} \sum_{k_{1}=3}^{N_{x}-2} \sum_{k_{2}=3}^{N_{y}-2} \gamma_{k} \bar{\beta}_{k} W_{k_{1}, k_{2}}, \\
W_{2, N_{y}-1}=\frac{1}{\alpha_{0} \bar{\alpha}_{0}} \sum_{k_{1}=3}^{N_{x}-2} \sum_{k_{2}=3}^{N_{y}-2} \beta_{k} \bar{\gamma}_{k} W_{k_{1}, k_{2}}, \\
W_{N_{x}-1, N_{y}-1}=\frac{1}{\alpha_{0} \bar{\alpha}_{0}} \sum_{k_{1}=3}^{N_{x}-2} \sum_{k_{2}=3}^{N_{y}-2} \gamma_{k} \bar{\gamma}_{k} W_{k_{1}, k_{2}} .
\end{array}
$$

Hence, the discretized governing equation (21) should be applied for the interior mesh points in which $3 \leq i \leq N_{x}-2$, $3 \leq j \leq N_{y}-2$.

Applying the discretized governing equation (21) for all the interior grid points (i.e., $3 \leq i \leq N_{x}-2,3 \leq j \leq$ $N_{y}-2$ ) and satisfying the boundary conditions (23a) and (23b) together with (25)-(27) will result in a set of algebraic equations in terms of $W_{i j}$. These equations can be expressed in the form of a matrix equation as follows:

$$
\mathbf{A W}-S_{\mathrm{cr}} \mathbf{B W}=\mathbf{0} \text {, }
$$


where $\mathbf{A}$ and $\mathbf{B}$ are two matrices of the coefficients, and $\mathbf{W}$ is the deflection vector in terms of $W_{i, j}$. Also, $S_{\mathrm{cr}}$ is the critical shear load per length $\left(S_{x y, \text { cr }}\right)$. Generally, the set of algebraic equations (28) should be of order $\left(N_{x} \times N_{y}\right)$ by $\left(N_{x} \times N_{y}\right)$. However, the use of (25) and (26) for $W_{2, j}, W_{N_{x}-1, j}, W_{i, 2}$, $W_{i, N_{y}-1}$ and (27) for $W_{2,2}, W_{N_{x}-1,2}, W_{2, N_{y}-1}$ and $W_{N_{x}-1, N_{y}-1}$ reduces the order of the algebraic equations to $\left[N_{x} \times N_{y}-\right.$ $\left.2\left(N_{x}+N_{y}\right)+12\right]$ by $\left[N_{x} \times N_{y}-2\left(N_{x}+N_{y}\right)+12\right]$.

By taking advantage of the reduced algebraic equations, the size of the analysis domain decreases and, consequently, a finer mesh can be used resulting in more accurate eigenvalues.

\section{Numerical Results and Discussion}

A mathematical code is developed according to the above described DQ solution to obtain the accurate critical buckling loads and their corresponding mode shapes for comparison studies. Also, some simulations were performed using ANSYS software. It should be pointed out that the assumptions of the classical plate theory (CPT) are incorporated into the simulations performed by ANSYS. The results obtained from the proposed closed-form formulas are compared to those obtained by the DQ code and ANSYS simulations to show the reliability of the formulas.

To generalize the numerical results, the following dimensionless parameters are introduced:

$$
\begin{aligned}
& k_{s}=\frac{b^{2}}{\pi^{2} D_{11}} S_{\mathrm{cr}}=\frac{b^{2} t}{\pi^{2} D_{11}} \tau_{\mathrm{cr}}, \\
& \bar{E}=\frac{E_{2}}{E_{1}}, \\
& \bar{G}=\frac{2\left(1+v_{12}\right)}{E_{1}} G_{12},
\end{aligned}
$$

where $k_{s}$ is dimensionless shear buckling parameter in terms of the critical load $S_{\text {cr }}\left(S_{x y, \text { cr }}\right)$ and the corresponding critical shear stress $\tau_{\mathrm{cr}}, t$ is the thickness, and $\bar{E}$ and $\bar{G}$ are dimensionless elasticity and shear moduli of orthotropic material, respectively. As a special case, the orthotropic plate is converted to an isotropic one when $\bar{E}=\bar{G}=1$.

Table 1 shows a convergence study of the DQ code as well as comparison of the dimensionless shear buckling parameter $k_{s}$ obtained from the closed-form approach, DQ solution, and those from [1] for fully clamped isotropic plates. The obtained buckling parameters are compared for various values of the aspect ratio $(\eta=a / b)$ and two different types of buckling modes: symmetric and antisymmetric. It can be observed from Table 1 that the obtained critical loads from both closed-form formulas and the DQ solution are in a very good agreement with the results of [1] for different values of the aspect ratio. Also, the results of this table confirm the convergence and stability of the obtained critical loads for all cases.

Variations of dimensionless fundamental shear buckling parameter $k_{s}$ versus the plate aspect ratio are depicted in Figure 3 for different values of the dimensionless material properties: $\bar{E}$ and $\bar{G}$. It can be seen from Figure 3 that

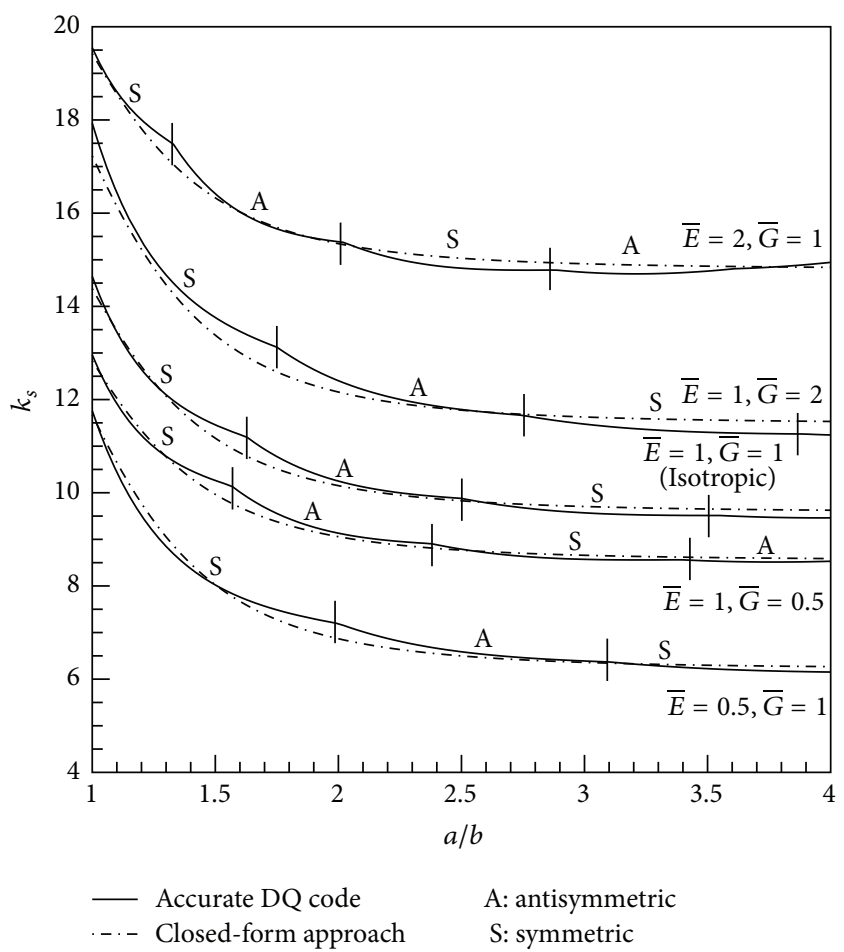

FIGURE 3: The critical buckling load coefficient for rectangular orthotropic plates under pure uniform shear load versus the aspect ratio for different material properties $\left(\nu_{21}=0.3\right)$.

the results of the proposed closed-form approach are in very good agreement with those of the time-consuming computational DQ solution for all cases, even for square plates. It is worth noting that the introduced closed-form formulas only predict the fundamental critical loads, either symmetric or antisymmetric mode. Although the plate aspect ratio influences the type of fundamental shear buckling mode, their critical loads are very close to each other. Since the curves of this figure are provided in dimensionless form, they can be used for estimating the critical shear loads of clamped orthotropic plates with a wide range of geometric and material properties.

To study the shear buckling of clamped narrow rectangular orthotropic plates, a special engineering panel is considered called laminated veneer lumber (LVL). This timber sheathing, with the commercial name of Kerto-Q, is subjected to a distributed uniform shear load. The mechanical and geometric properties of LVL are as follows:

$$
\begin{aligned}
E_{1} & =10.5 \mathrm{GPa}, \\
E_{2} & =2.4 \mathrm{GPa}, \\
G_{12} & =0.6 \mathrm{GPa}, \\
v_{21} & =0.05, \\
a & =3.0 \mathrm{~m}, \\
b & =0.7275 \mathrm{~m}, \\
t & =0.027 \mathrm{~m} .
\end{aligned}
$$


TABLE 1: Convergence study and comparison of critical buckling load parameter $\left(k_{s}\right)$ of fully clamped isotropic rectangular plates with various values of the aspect ratio $(\eta=a / b)$ under uniform distributed shear load.

\begin{tabular}{|c|c|c|c|c|c|c|c|c|c|c|}
\hline \multirow{3}{*}{$N_{x} \times N_{y}$} & \multicolumn{10}{|c|}{$k_{s}=b^{2} S_{\mathrm{cr}} /\left(\pi^{2} D_{11}\right)$} \\
\hline & \multicolumn{2}{|c|}{$\eta=1.00$} & \multicolumn{2}{|c|}{$\eta=1.25$} & \multicolumn{2}{|c|}{$\eta=1.50$} & \multicolumn{2}{|c|}{$\eta=2.00$} & \multicolumn{2}{|c|}{$\eta=3.00$} \\
\hline & Sym. & Antisym. & Sym. & Antisym. & Sym. & Antisym. & Sym. & Antisym. & Sym. & Antisym. \\
\hline $7 \times 7$ & 14.938 & 16.335 & 12.543 & 13.488 & 11.619 & 12.129 & 11.358 & 10.618 & 12.696 & 12.901 \\
\hline $9 \times 9$ & 14.801 & 17.307 & 12.487 & 13.979 & 11.602 & 12.056 & 10.774 & 10.418 & 9.816 & 10.005 \\
\hline $11 \times 11$ & 14.643 & 16.904 & 12.347 & 13.658 & 11.457 & 11.797 & 10.579 & 10.251 & 9.568 & 9.709 \\
\hline $13 \times 13$ & 14.642 & 16.920 & 12.347 & 13.671 & 11.459 & 11.805 & 10.583 & 10.249 & 9.535 & 9.626 \\
\hline $15 \times 15$ & 14.642 & 16.919 & 12.347 & 13.670 & 11.458 & 11.804 & 10.582 & 10.248 & 9.568 & 9.632 \\
\hline $17 \times 17$ & 14.642 & 16.919 & 12.347 & 13.670 & 11.458 & 11.804 & 10.582 & 10.248 & 9.569 & 9.631 \\
\hline Closed-form approach & 14.382 & - & 12.374 & - & 11.170 & - & - & 10.152 & 9.705 & - \\
\hline Reference [1] & 14.64 & - & 12.35 & - & 11.45 & 11.79 & 10.58 & 10.32 & 9.57 & 9.64 \\
\hline
\end{tabular}

TABLE 2: Convergence study and comparison of critical buckling shear stress, $\tau_{\mathrm{cr}}(\mathrm{MPa})$, of fully clamped orthotropic rectangular plate (LVL) with FEM simulation and the closed-form approach.

\begin{tabular}{lcc}
\hline \multirow{2}{*}{$N_{x} \times N_{y}$} & \multicolumn{2}{c}{$\tau_{\text {cr }}(\mathrm{MPa})$} \\
\hline $7 \times 7$ & 42.788 & 43.701 \\
$9 \times 9$ & 31.421 & 32.410 \\
$11 \times 11$ & 30.484 & 31.107 \\
$13 \times 13$ & 30.321 & 30.582 \\
$15 \times 15$ & 30.334 & 30.623 \\
$17 \times 17$ & 30.331 & 30.617 \\
$19 \times 19$ & 30.332 & 30.618 \\
Closed-form approach & 30.534 & - \\
ANSYS simulation & 30.410 & 30.694 \\
\hline
\end{tabular}

In order to ensure convergence and accuracy of the developed DQ code for the shear buckling loads of the orthotropic narrow plates, a convergence study is presented in Table 2 for the LVL sheathing. Also, the results are compared with those obtained from an accurate FE simulation via ANSYS software as well as the results of the closed-form approach. It is evident that the obtained critical in-plane shear stresses from the closed-form formulas are in very good agreement with those achieved from the DQ code and ANSYS simulation.

In order to get a better physical sense of the symmetric and antisymmetric modes, the mode shapes of a square isotropic clamped plate are provided via both the developed DQ code and ANSYS simulation and are illustrated in Figure 4. Also, the symmetric and antisymmetric mode shapes of the LVL narrow rectangular plate are presented in Figure 5 to show the influence of the geometric shape on the mode shapes. It can be seen that the mode shapes obtained by the DQ code are the same as those obtained by ANSYS simulation. Also, comparison between the corresponding mode shapes of Figures 4 and 5 reveals the fact that the number of half-waves increases by increasing the aspect ratio.

In Figure 6, the mode shape of the LVL obtained from the closed-form approximation is shown. It can be observed that the mode shape predicted by this approximate method, except at the two short edges, is similar to those of other methods.

Variations of the in-plane shear stress of the clamped LVL plate versus different geometric and mechanical parameters are shown in Figures 7 and 8, respectively. It should be pointed out that the results in these two figures are presented in dimensional form to more directly study the influence of various parameters on the critical in-plane shear stress. In both figures, the results of DQ code, closed-form approach and FE simulation via ANSYS are provided to show the reliability and efficiency of the developed closedform approach for a wide variety of different geometric and material properties. In Figure $7, \lambda$ denotes one of the geometric properties relative to the reference value for the LVL panel and in Figure $8 \gamma$ represents one of the material properties. The subscript "LVL" in the relative expressions $\lambda_{i} / \lambda_{\mathrm{LVL}}$ and $\gamma_{i} / \gamma_{\mathrm{LVL}}$ refers to the reference values of the LVL panel (see (30)). It can be observed from Figure 7 that the critical buckling stress considerably decreases as the width $b$ of the LVL plate increases, whereas decreasing the length $a$ results in a small increase of the critical buckling stress. Also, it is evident that the critical stress significantly increases when the thickness of the plate increases. However, it should be mentioned that for large thicknesses the classical plate theory is no longer valid due to the neglect of the transverse shear deformations.

Figure 8 reveals the fact that, by increasing any of the material properties, the critical buckling stress increases. It is also obvious that Young's modulus $E_{2}$ has the largest effect and the shear modulus $G_{12}$ the smallest effect on the critical buckling stress of the clamped narrow rectangular orthotropic plate subjected to uniform in-plane shear load.

Influence of Poisson's ratio $v_{21}$ on the critical in-plane shear stress of the clamped LVL is shown in Figure 9 based on the obtained closed-form formulas, DQ solution, and ANSYS simulations. It can be observed that increasing Poisson's ratio can slightly increase the critical shear stress.

In Figures 7-9, very good agreement between the curves obtained from the closed-form approach and those based on the DQ code as well as ANSYS simulation shows the accuracy and reliability of the proposed efficient closed-form formula for all cases. 


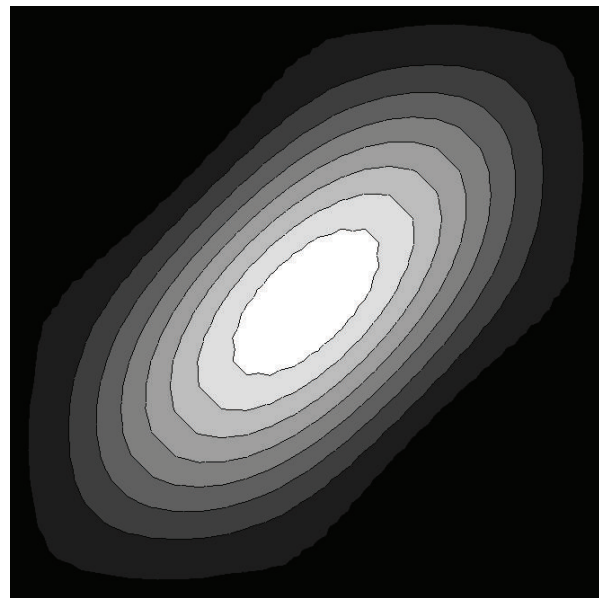

DQ code

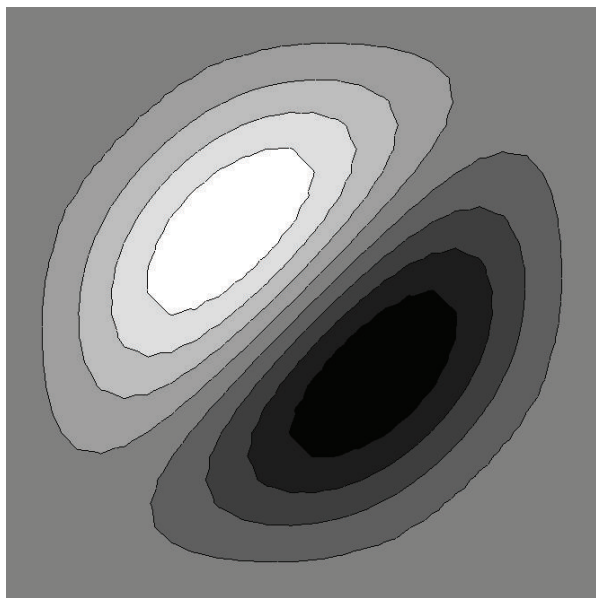

DQ code

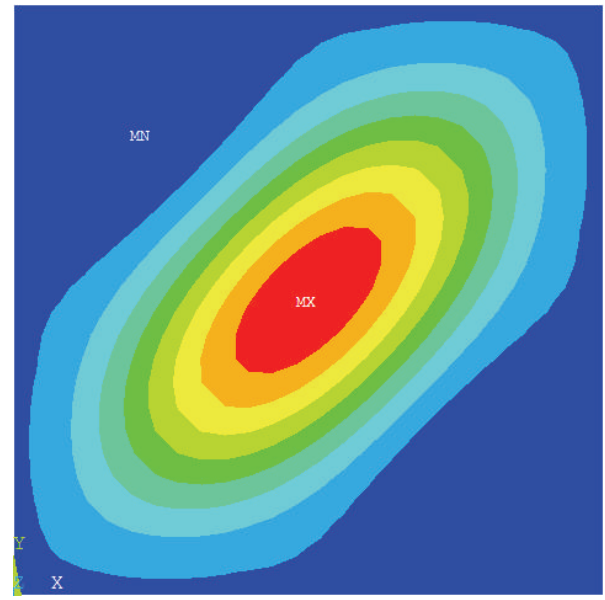

ANSYS simulation

(a)

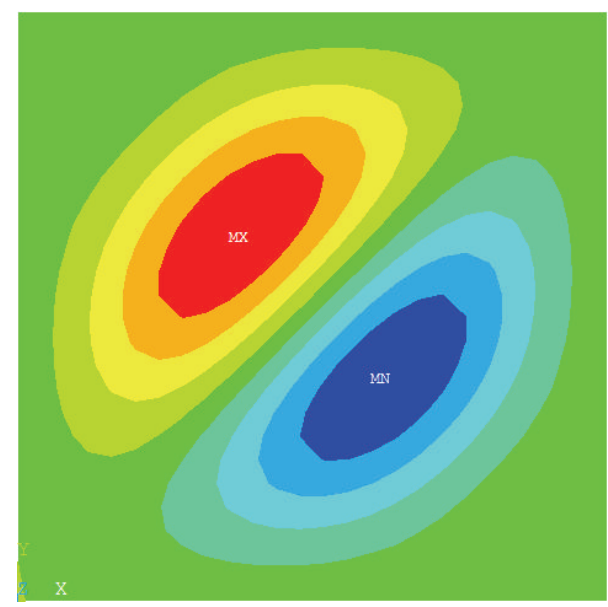

ANSYS simulation

(b)

FIGURE 4: Contour plot of the mode shape corresponding to the critical buckling loads of a fully clamped isotropic square plate: (a) symmetric mode, (b) antisymmetric mode.

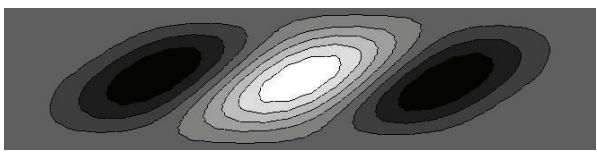

DQ code

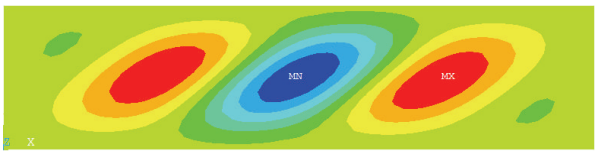

ANSYS simulation

(a)

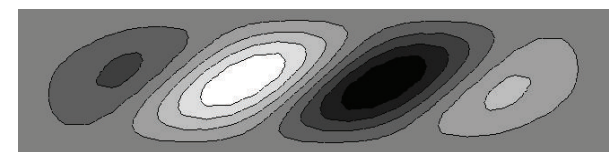

DQ code

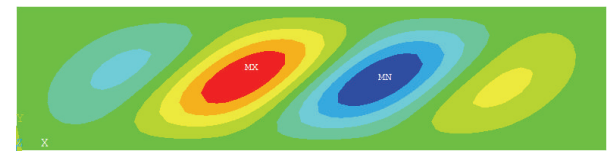

ANSYS simulation

(b)

FIGURE 5: Contour plot of the mode shape corresponding to the critical buckling loads of a fully clamped orthotropic rectangular plate (LVL): (a) symmetric mode, (b) antisymmetric mode. 


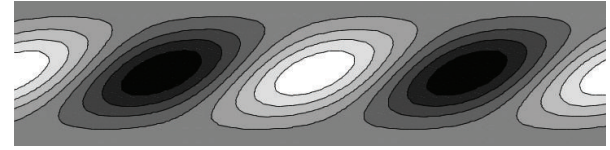

FIgURE 6: Contour plot of the mode shape corresponding to the critical buckling load of the orthotropic LVL plate based on the closed-form approach.

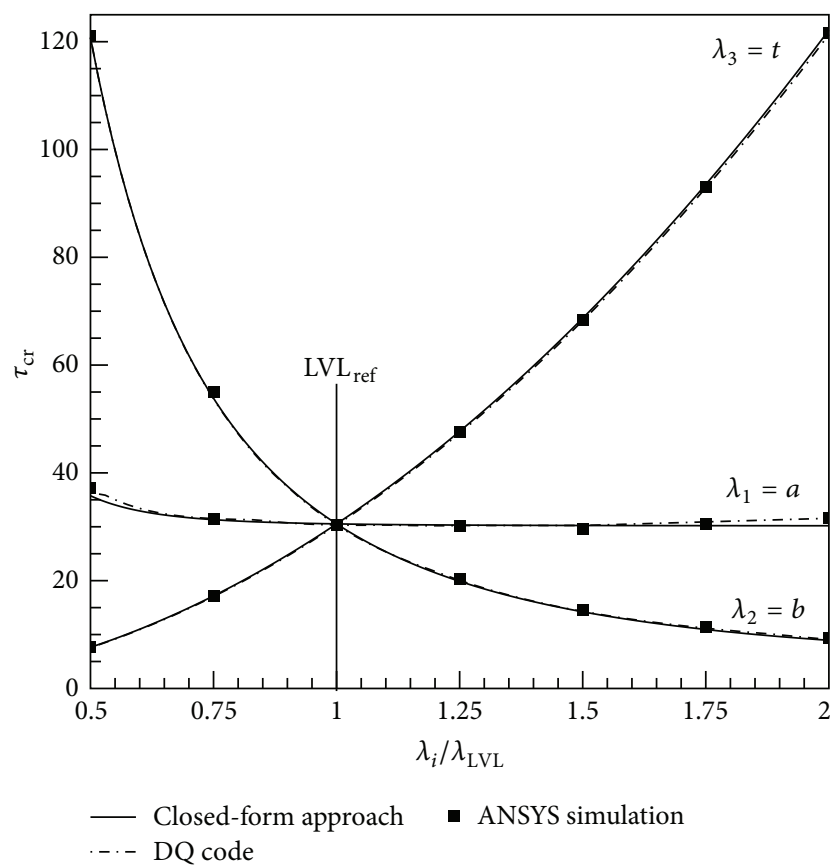

FiguRE 7: Effect of different geometric properties on the critical shear stress of the orthotropic LVL panel. The horizontal axis shows the relative value of the geometric parameter with reference to the value of the LVL panel.

\section{Conclusions}

In this paper, the shear buckling of clamped narrow rectangular orthotropic plates was investigated. An efficient closedform approach was presented to easily and fastly predict the critical shear buckling loads and corresponding mode-shapes of the clamped narrow rectangular orthotropic plates. Also, a practical modification factor was proposed to extend the validity range of the obtained explicit formulas. To prove the accuracy and effectiveness of the closed-form approach, an accurate DQ code was developed and the critical buckling loads and their corresponding mode shapes were extracted. Also, several accurate FE simulations using ANSYS software were performed. It was shown that the proposed closed-form approach can predict the critical buckling loads with the acceptable accuracy for a wide range of effective parameters without any computational effort. The effect of various geometric and mechanical parameters was investigated by means of three different methods: closed-form approach, DQ code, and ANSYS simulations. It was observed that the critical buckling load considerably decreases by increasing the width $b$ of the narrow plates whereas decreasing the length $a$ results

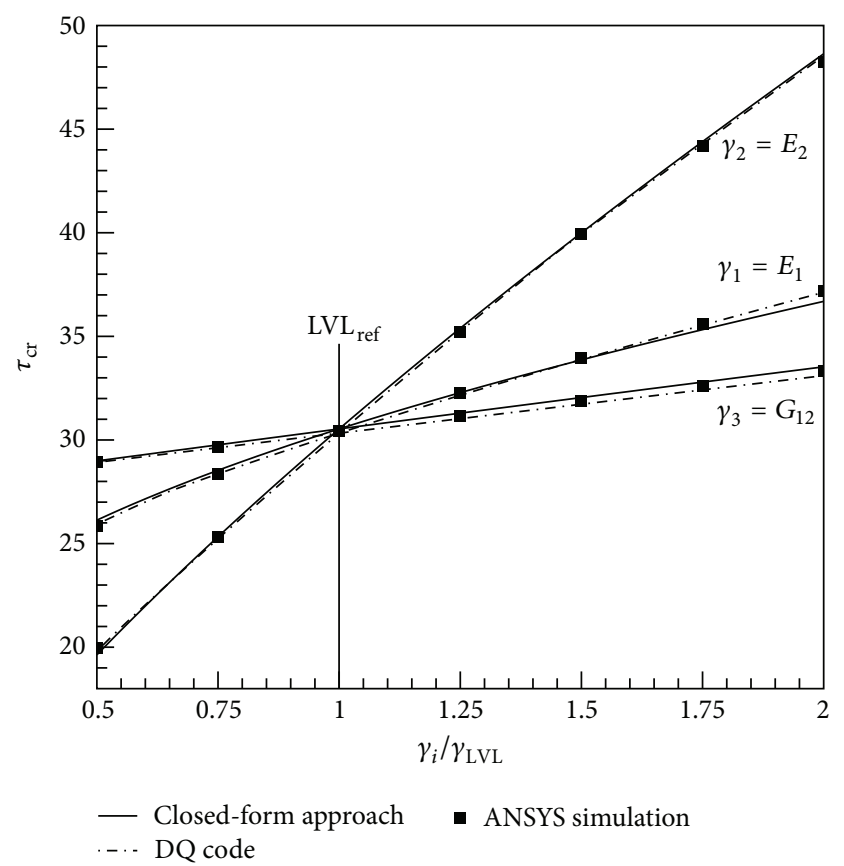

FIGURE 8: Effect of different material properties on the critical shear stress of the orthotropic LVL panel. The horizontal axis shows the relative value of the material parameter with reference to the value of the LVL panel.

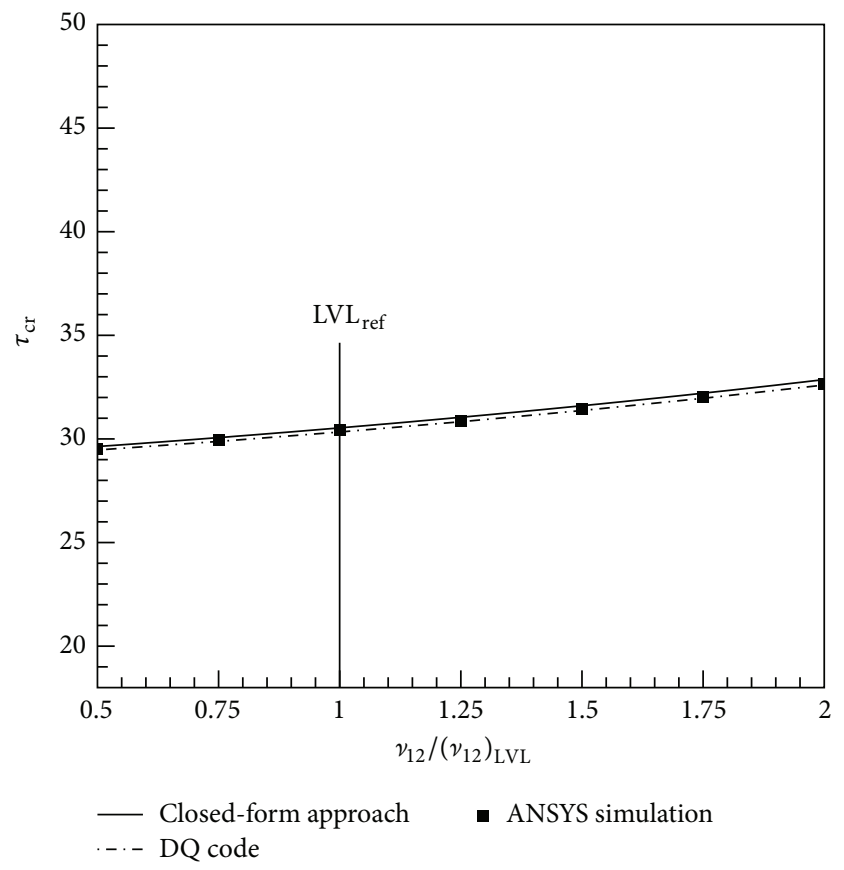

Figure 9: Effect of Poisson's ratio on the critical shear stress of the orthotropic LVL panel. The horizontal axis shows the relative value of the material parameter with reference to the value of the LVL panel. 
in a very small increase of the critical buckling load. Also it was shown, among different material properties, that Young's modulus $E_{2}$ and the shear modulus $G_{12}$ have the largest and smallest effects on the critical buckling load, respectively.

\section{Appendix}

\section{Calculation of Weighting and Other Coefficients}

The weighting coefficients for the first-order derivatives are expressed as

$$
\begin{aligned}
& c_{i j}^{(1)}=\frac{\alpha^{(1)}\left(x_{i}\right)}{\left(x_{i}-x_{j}\right) \alpha^{(1)}\left(x_{j}\right)}, \\
& c_{i i}^{(1)}=-\sum_{j=1, j \neq i}^{N_{x}} c_{i j}^{(1)} \\
& \bar{c}_{i j}^{(1)}=\frac{\beta^{(1)}\left(y_{i}\right)}{\left(y_{i}-y_{j}\right) \beta^{(1)}\left(y_{j}\right)}, \\
& \bar{c}_{i i}^{(1)}=-\sum_{j=1, j \neq i}^{N_{y}} \bar{c}_{i j}^{(1)}
\end{aligned}
$$

in which the functions $\alpha$ and $\beta$ are represented in the form

$$
\begin{aligned}
& \alpha^{(1)}\left(x_{i}\right)=\prod_{j=1, j \neq i}^{N_{x}}\left(x_{i}-x_{j}\right), \\
& \beta^{(1)}\left(y_{i}\right)=\prod_{j=1, j \neq i}^{N_{y}}\left(y_{i}-y_{j}\right) .
\end{aligned}
$$

The higher-order weighting coefficients are expressed by the following recursive relations:

$$
\begin{aligned}
c_{i j}^{(n)}= & n\left(c_{i j}^{(1)} c_{i i}^{(n-1)}-\frac{c_{i j}^{(n-1)}}{x_{i}-x_{j}}\right), \\
c_{i i}^{(n)}= & -\sum_{j=1, j \neq i}^{N_{x}} c_{i j}^{(n)} \\
& i, j=1,2, \ldots, N_{x} ; n=2,3, \ldots, N_{x}-1, i \neq j, \\
\bar{c}_{i j}^{(m)}= & m\left(\bar{c}_{i j}^{(1)} \bar{c}_{i i}^{(m-1)}-\frac{\bar{c}_{i j}^{(m-1)}}{y_{i}-y_{j}}\right), \\
\bar{c}_{i i}^{(m)}= & -\sum_{j=1, j \neq i}^{N_{y}} \bar{c}_{i j}^{(m)} \\
& i, j=1,2, \ldots, N_{y} ; m=2,3, \ldots, N_{y}-1, i \neq j .
\end{aligned}
$$

The coefficients $\alpha_{0}, \beta_{k}, \gamma_{k}, \bar{\alpha}_{0}, \bar{\beta}_{k}$, and $\bar{\gamma}_{k}$, in (25) and (26), are defined as

$$
\begin{aligned}
& \alpha_{0}=c_{N_{x}, 2}^{(1)} \cdot c_{1, N_{x}-1}^{(1)}-c_{1,2}^{(1)} \cdot c_{N_{x}, N_{x}-1}^{(1)}, \\
& \beta_{k}=c_{1, k}^{(1)} \cdot c_{N_{x}, N_{x}-1}^{(1)}-c_{1, N_{x}-1}^{(1)} \cdot c_{N_{x}, k}^{(1)}, \\
& \gamma_{k}=c_{1,2}^{(1)} \cdot c_{N_{x}, k}^{(1)}-c_{1, k}^{(1)} \cdot c_{N_{x}, 2}^{(1)}, \\
& \bar{\alpha}_{0}=\bar{c}_{N_{y}, 2}^{(1)} \cdot \bar{c}_{1, N_{y}-1}^{(1)}-\bar{c}_{1,2}^{(1)} \cdot \bar{c}_{N_{y}, N_{y}-1}^{(1)}, \\
& \bar{\beta}_{k}=\bar{c}_{1, k}^{(1)} \cdot \bar{c}_{N_{y}, N_{y}-1}^{(1)}-\bar{c}_{1, N_{y}-1}^{(1)} \cdot \bar{c}_{N_{y}, k}^{(1)}, \\
& \bar{\gamma}_{k}=\bar{c}_{1,2}^{(1)} \cdot \bar{c}_{N_{y}, k}^{(1)}-\bar{c}_{1, k}^{(1)} \cdot \bar{c}_{N_{y}, 2}^{(1)} .
\end{aligned}
$$

\section{Conflict of Interests}

The authors declare that there is no conflict of interests regarding the publication of this paper.

\section{Acknowledgments}

The authors would like to express their sincere appreciation for the financial support from the Regional Council of Västerbotten, the County Administrative Board in Norrbotten, and The European Union's Structural Funds, The Regional Fund.

\section{References}

[1] B. Budiansky and R. W. Connor, "Buckling stresses of clamped rectangular flat plates in shear," Tech Note 1559, NACA, 1948.

[2] D. J. Johns, "Shear buckling of isotropic and orthotropic plates: a review," Aeronautical Research Council Report, Ministry of Defense, HMSO, London, UK, 1971.

[3] D. Hui, "Shear buckling of anti-symmetric cross ply rectangular plates," Fibre Science and Technology, vol. 21, no. 4, pp. 327-340, 1984.

[4] S. Kosteletos, "Shear buckling response of laminated plates," Composite Structures, vol. 20, no. 3, pp. 147-154, 1992.

[5] S. B. Biggers and S. S. Pageau, "Shear buckling response of tailored composite plates," AIAA Journal, vol. 32, no. 5, pp. 11001103, 1994.

[6] Y. Xiang, C. M. Wang, and S. Kitipornchai, "Shear buckling of simply supported skew mindlin plates," AIAA Journal, vol. 33, no. 2, pp. 377-378, 1995.

[7] J. Loughlan, “The shear buckling behaviour of thin composite plates with particular reference to the effects of bend-twist coupling," International Journal of Mechanical Sciences, vol. 43, no. 3, pp. 771-792, 2001.

[8] A. V. Lopatin and Y. B. Korbut, "Buckling of clamped orthotropic plate in shear," Composite Structures, vol. 76, no. 1-2, pp. 94-98, 2006.

[9] I. Shufrin and M. Eisenberger, "Shear buckling of thin plates with constant in-plane stresses," International Journal of Structural Stability and Dynamics, vol. 7, no. 2, pp. 179-192, 2007.

[10] L. Papadopoulos and C. Kassapoglou, "Shear buckling of rectangular composite plates composed of concentric layups," 
Composites Part A: Applied Science and Manufacturing, vol. 38, no. 5, pp. 1425-1430, 2007.

[11] L.-Y. Wu, C.-H. Wu, and H.-H. Huang, "Shear buckling of thin plates using the spline collocation method," International Journal of Structural Stability and Dynamics, vol. 8, no. 4, pp. 645-664, 2008.

[12] B. Uymaz and M. Aydogdu, "Three dimensional shear buckling of FG plates with various boundary conditions," Composite Structures, vol. 96, pp. 670-682, 2013.

[13] M. Shariyat and K. Asemi, "Three-dimensional non-linear elasticity-based 3D cubic B-spline finite element shear buckling analysis of rectangular orthotropic FGM plates surrounded by elastic foundations," Composites Part B: Engineering, vol. 56, pp. 934-947, 2014.

[14] S. P. Timoshenko and J. M. Gere, Theory of Elastic Stability, International Student Edition, McGraw-Hill, 2nd edition, 1961.

[15] Ö. Civalek, "Application of differential quadrature (DQ) and harmonic differential quadrature (HDQ) for buckling analysis of thin isotropic plates and elastic columns," Engineering Structures, vol. 26, no. 2, pp. 171-186, 2004.

[16] A. N. Sherbourne and M. D. Pandey, "Differential quadrature method in the buckling analysis of beams and composite plates," Computers and Structures, vol. 40, no. 4, pp. 903-913, 1991.

[17] Ö. Civalek, "Free vibration and buckling analyses of composite plates with straight-sided quadrilateral domain based on DSC approach," Finite Elements in Analysis and Design, vol. 43, no. 13, pp. 1013-1022, 2007.

[18] X. Wang, L. Gan, and Y. Zhang, "Differential quadrature analysis of the buckling of thin rectangular plates with cosinedistributed compressive loads on two opposite sides," Advances in Engineering Software, vol. 39, no. 6, pp. 497-504, 2008.

[19] C. Shu, Differential Quadrature and Its Application in Engineering, Springer, London, UK, 2000.

[20] C. Shu and H. Du, "Implementation of clamped and simply supported boundary conditions in the GDQ free vibration analysis of beams and plates," International Journal of Solids and Structures, vol. 34, no. 7, pp. 819-835, 1997. 


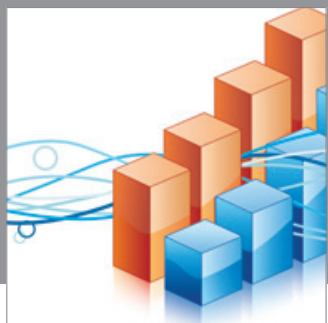

Advances in

Operations Research

mansans

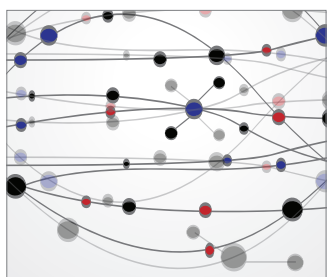

The Scientific World Journal
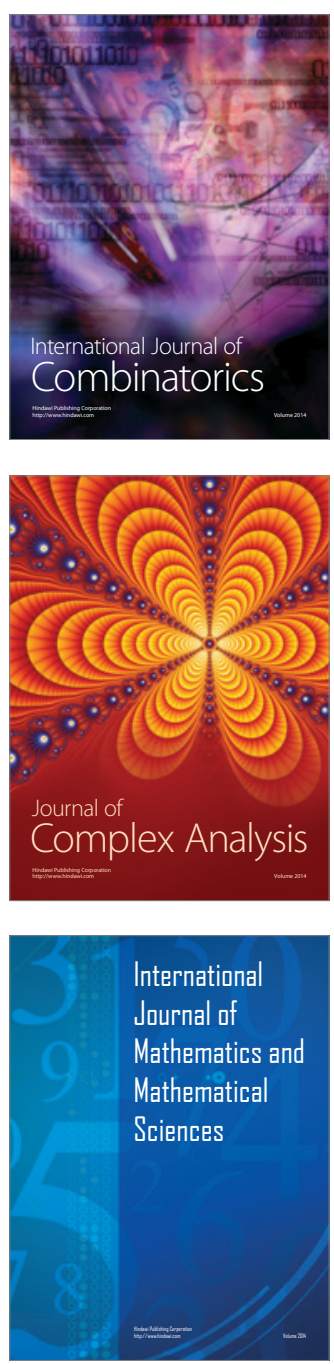
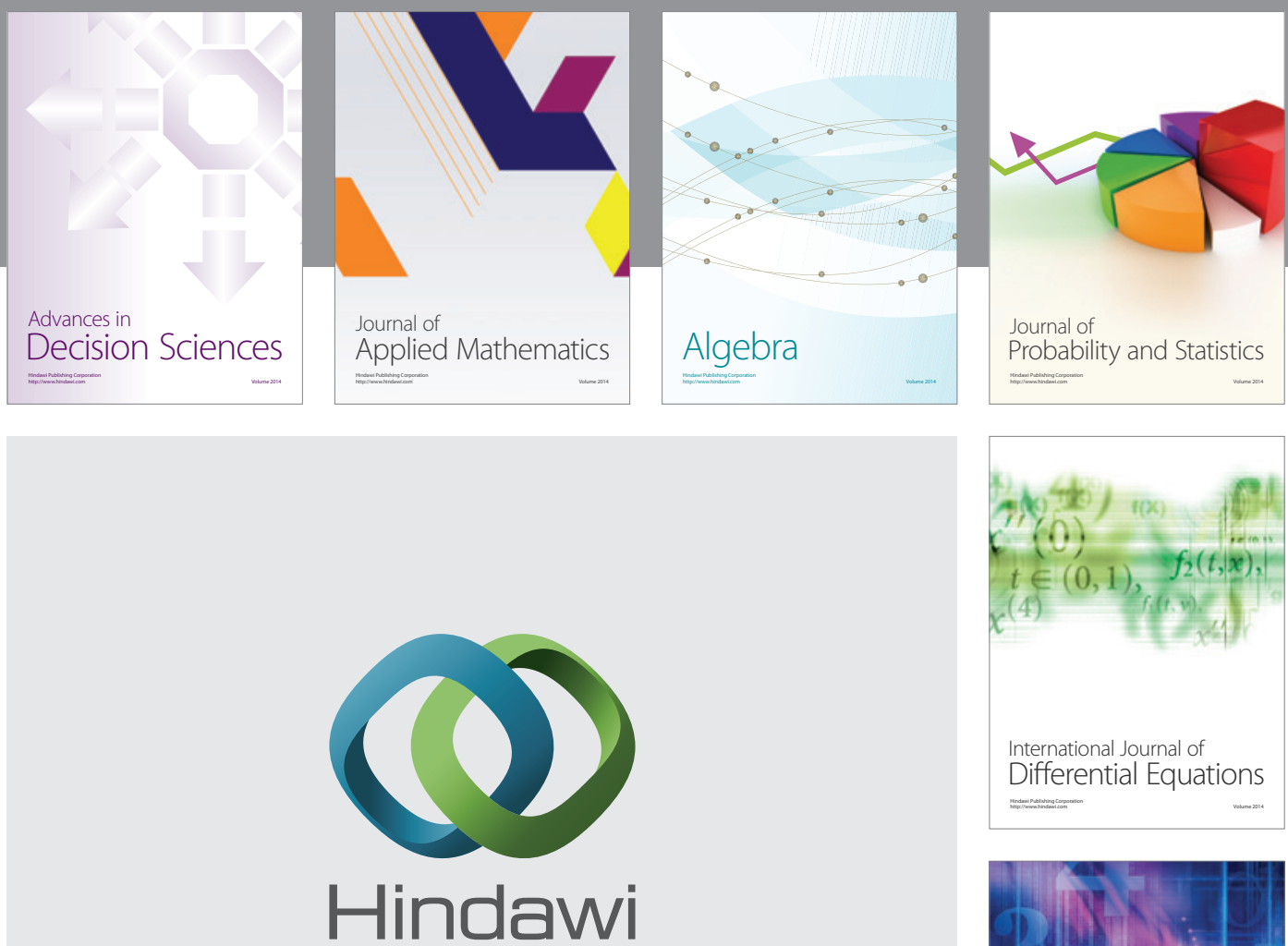

Submit your manuscripts at http://www.hindawi.com
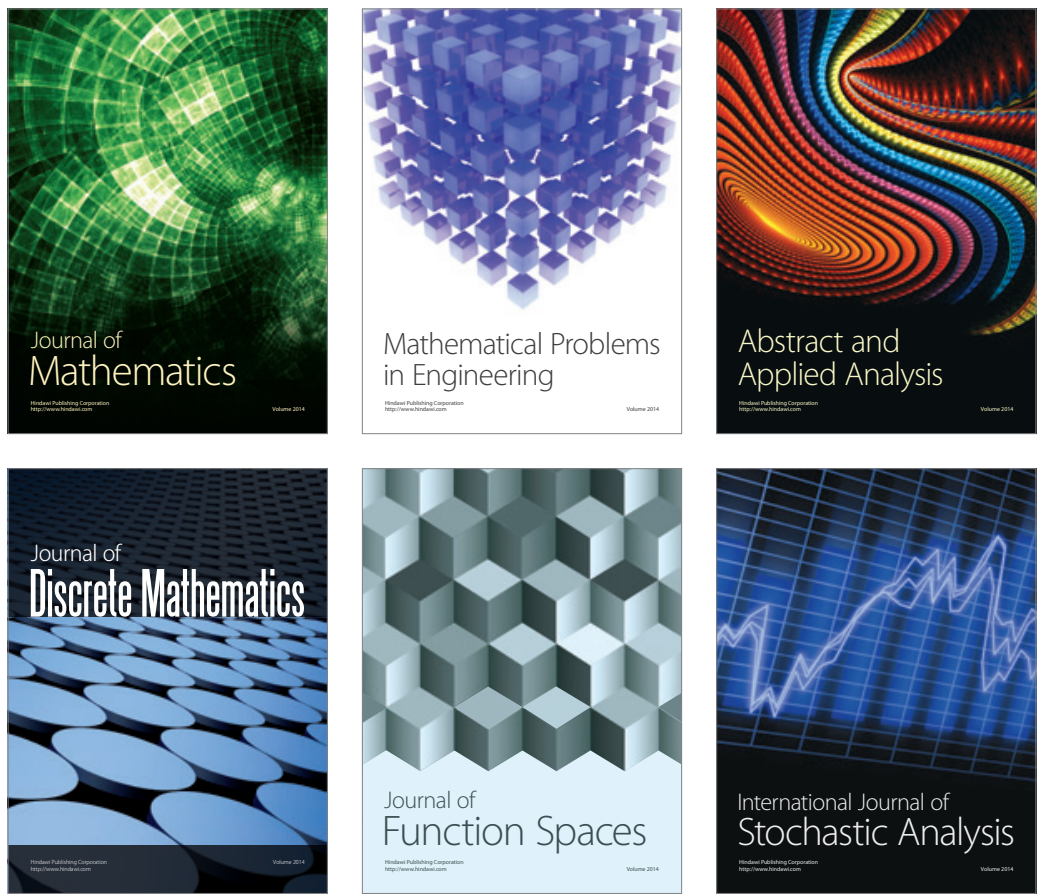

Journal of

Function Spaces

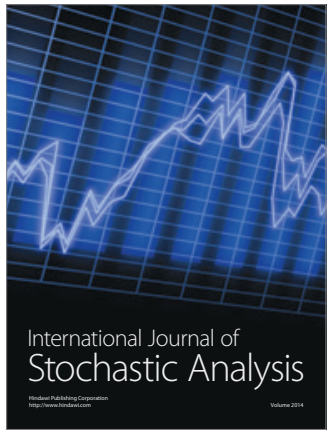

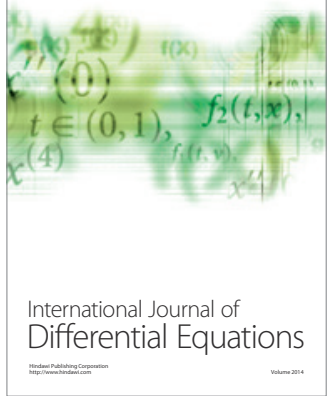
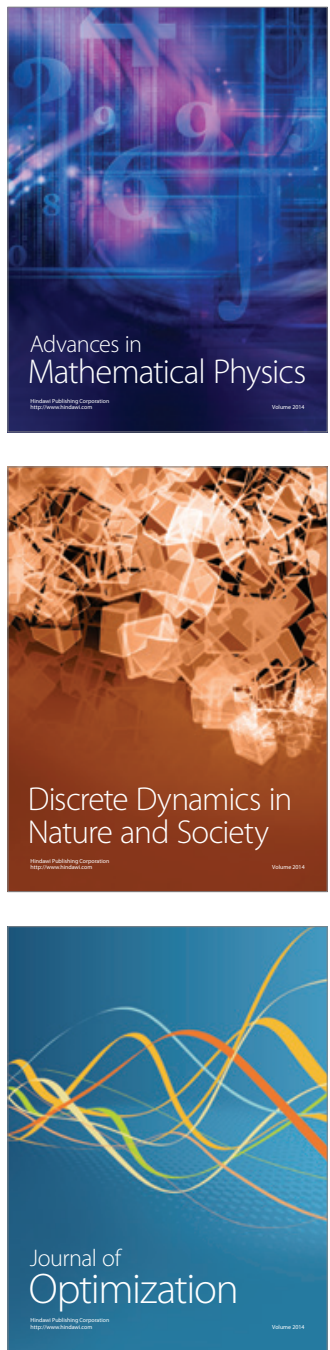